\title{
The Spin-Orbit Evolution of GJ 667C System: The Effect of Composition and Other Planet's Perturbations
}

\author{
P.A. Cuartas-Restrepo ${ }^{1 \star}$, M. Melita ${ }^{2}$, J.I. Zuluaga ${ }^{1}$, \\ B. Portilla-Revelo ${ }^{1}$, M. Sucerquia ${ }^{1}$, O. Miloni ${ }^{3}$. \\ ${ }^{1}$ FACom - SEAP - Instituto de Física - FCEN, Universidad de Antioquia, Calle 70 No. 52-21, Medellín, Colombia. \\ ${ }^{2}$ Instituto de Astronomía y Física del Espacio - IAFE, Buenos Aires, Argentina. \\ ${ }^{3}$ FCAG - Universidad Nacional de La Plata, La Plata, Argentina.
}

Released 2002 Xxxxx XX

\begin{abstract}
Potentially habitable planets within the habitable zone of M-dwarfs are affected by tidal interaction. We studied the tidal evolution in GJ $667 \mathrm{C}$ using a numerical code we call TIDEV. We reviewed the problem of the dynamical evolution focusing on the effects that a rheological treatment, different compositions and the inclusion of orbital perturbations, have on the spin-down time and the probability to be trapped in a low spin-orbit resonance. Composition have a noticiable effect on the spin-down time, changing, in some cases, by almost a factor of 2 with respect to the value estimated for a reference Earth-like model. We calculated the time to reach a low resonance value $(3: 2)$ for the configuration of 6 planets. Capture probabilities are affected when assuming different compositions and eccentricities variations. We chose planets $b$ and c to evaluate the probabilities of capture in resonances below 5:2 for two compositions: Earth-like and Waterworld planets. We found that perturbations, although having a secular effect on eccentricities, have a low impact on capture probabilities and nothing on spin-down times. The implications of the eccentricity variations and actual habitability of the GJ $667 \mathrm{C}$ system are discussed.
\end{abstract}

Key words: Planet-star interactions - Planets and satellites: dynamical evolution, individual: GJ 667C.

\section{INTRODUCTION}

Finding a habitable Earth-like planet has become the holy grail of exoplanetary research. Planetary habitability is a complex property constrained by factors ranging from stellar insolation and the characteristics of planetary atmosphere to interior structure, magnetic field strength and rate and direction of planetary rotation (Lammer et al. 2010).

The evaluation of a few of these factors on true exoplanets is relatively straight forward (e.g. stellar insolation or planetary gravity), but others (e.g. planetary magnetic fields and rotation), remain elusive and are still a matter of investigation (Zuluaga \& Cuartas 2012, Zuluaga et al. 2013 ).

In the case of low mass stars (M-dwarfs or dMs), the Habitable Zone (HZ) is close to the star. Under this condition, tidal interactions can be modeled to estimate the final

\footnotetext{
* E-mail: pablo.cuartas@udea.edu.co
}

rotational state of nearby planets. From there, it is possible to constrain other properties that depend on rotation, which could affect habitability, like intrinsic magnetic fields (Zuluaga \& Cuartas 2012; Zuluaga et al. 2013).

Spin-orbit evolution of Earth-like exoplanets and superEarths, like planets in the GJ $667 \mathrm{C}$ system, have been a matter of research in the last couple of years (Heller et al. 2011; Rodríguez et al. 2012, Callegari \& Rodríguez 2013. Anglada-Escudé et al. 2013). Most of these approaches have used a traditional tidal formalism where torques are frequency independent, neglecting the complexity of planetary rheology (Correia et al. 2008, Ferraz-Mello et al. 2008, Leconte et al. 2010).

The dependence on the rheology remains an open discussion. Recently, Ferraz-Mello (2013) and Correia et al. (2014) reviewed the problem of the tidal torque; they found that for rocky bodies, independent of the eccentricity, the stationary rotation is close to the resonance. The second work by Correia et al. (2014) assumes a Maxwell's classic 
viscoelastic rheology to calculate the body deformation and applies their model to already discovered super-Earths. For this kind of planets, they found that the rotation stops temporarily in synchronous resonances during the spin-orbit evolution. Eventually, these stationary stages are crossed as long as the eccentricity decreases. They suggest that in the presence of other planets in the system, high order resonances could remain during all the planetary evolution. They conclude that close-in Earth-like planets could be captured in non-synchronous spin-orbit resonances.

On the other hand, another approach to the problem was developed and applied successfully by Efroimsky, Makarov \& Williams in their works (Efroimsky \& Williams 2009 |Makarov \& Efroimsky 2013;| Efroimsky| 2012;| Makarov 2012 Williams \& Efroimsky 2012 Makarov \& Berghea 2014 Makarov 2015). This model, based on the seed work by Darwin (1879) and developed later by Kaula (1964), treats realistically the dependency on rheology, and hence, on frequency of the tidal torque.

In this work we applied the Efroimsky-MakarovWilliams treatment (hereafter EMW) included numerically in a package developed by ourselves that we call Tidal Evolution Package - TIDEV, to analize the tidal interactions and spin-orbit evolution in the GJ $667 \mathrm{C}$ planetary system. Although Makarov \& Berghea (2014) recently applied the same formalism to study this planetary system, their approach neglected the possible existence of other planets around GJ 667C, announced by Anglada-Escudé et al. (2013). We analyzed three possible configurations for the planetary system, including the possible configuration of 2 and 3 planets, recently announced by Feroz \& Hobson (2014). For our study purposes, we used the main physical and orbital parameters of the system that has been updated by Anglada-Escudé et al. (2013) (see Table 1).

Regarding numerical codes devoted to the evolution of tides in compact planetary systems, the recent work of Bolmont et al. (2015) proposes a tool based on the well-known code Mercury, to which they have added the equations of tidal torque and other aspects as the relativistic effects and the deformation of the bodies. They have named their code Mercury-T. This work includes a classic treatment of the tidal evolution problem using a model of constant delay (constant lag) without averaged equations, which allows them to calculate the crossings through resonances and the probabilities of tidal locking. Nevertheless, they do not include the gravitational interaction between other possible members of the system. In Section 4 , we do a more detailed comparison with our own code of tidal evolution.

Our aim in this work is to study the impact that planetary composition could have on the tidal evolution of a multiplanetary system and its final rotational state, regardless of the number of members of the system. In the case of GJ $667 \mathrm{C}$, being a dynamically-packed system, the effect of orbital perturbations could also be important when studying the tidal interaction and its evolution. Here, we present and apply the EMW approach and include the effect of secular perturbations in the integration of tidal evolution equations using TIDEV, that includes the tidal equations proposed mainly in the works by Efroimsky \& Williams (2009), Efroimsky (2012), Makarov \& Efroimsky (2013) and Efroimsky \& Makarov (2013).

This paper is organized as follows: Section 2 describes the main properties of the GJ $667 \mathrm{C}$ system including a complete analysis of the secular evolution of three different configurations: 2, 3 and 6 planets. In section 3 we describe the basic theory and formalism of tidal torque proposed by EMW. Section 4 explains our theoretical model, our numerical experiments and the analysis of the effect of the other members of the planetary system over the dynamical evolution of planets $b$ and $c$. Section 5 shows our main results. Finally, in Section 6 we discuss the results and talk about the actual habitability of GJ $667 \mathrm{C}$.

\section{THE GJ 667C SYSTEM}

GJ $667 \mathrm{C}$ is a $\mathrm{M} 1.5 \mathrm{~V}, 0.33 M_{\odot}$ member of a triple stellar system, located at $6.8 \mathrm{pc}$ from the Earth in the direction of Scorpius. The star is in a wide orbit $\sim 230$ AU away from the center of mass of two close packed companions, GJ 667A and GJ 667B (Anglada-Escudé et al. 2013). Spectroscopic studies suggest that the main binary members (AB) are main sequence stars with an estimated age larger than 2 Gyr (Cayrel de Strobel 1981). Independently, the presentday measured rotational period of GJ $667 \mathrm{C}(\sim 100$ days $)$ and its signals of chromospheric activity points out to an age $\sim 8$ Gyr (Anglada-Escudé et al. 2013).

The GJ 667 system has recently been a cause of debate because of the actual number of planets orbiting the C star. Lately, It has been shown that this planetary system has at least two planets, one of them (planet c) within the HZ (Anglada-Escudé et al. 2012, 2013 Feroz \& Hobson 2014). The configuration proposed by Anglada-Escudé et al. (2013) is 6 planets closely packed in orbits with semimajor axes between 0.05 and 0.5 AU. Table 1 summarizes the basic physical and orbital properties of the system as reported by Anglada-Escudé et al. (2013). In their work, they propose the possible existence of a seventh planet with an orbit among the already announced members. The observed mass of the planets, between 1.94-5.94 $M_{\oplus}$, locate them in the mass range of super-Earths. However, and according to Kepler's most recent results, there is a significant chance that at least the more massive members of the system could actually be mini Neptunes, volatile rich planets (Benneke \& Seager 2013). Planetary formation theories suggest that this kind of planets could be water rich and hence, if solid, true Waterworlds (Kaltenegger et al. 2013).

On the other hand, a more recent work by Feroz \& Hobson (2014) proposed a more simple configuration including only two planets, both confirmed using a bayesian analysis of the radial velocity observations. In the same work they propose the possibility of a third signal (another planet) with a period of $91 \mathrm{~d}$. We include this possibility as a true planet.

For our purpose, the three possible configurations are suitable to be anlyzed dynamically. Our aim is to understand the spin-orbit evolution of a closely packed planetary system, regardless of the existing number of planets. We have a special interest on the GJ $667 \mathrm{C}$ system due to the potential habitability of planet $\mathrm{c}$.

The inclusion of secular perturbations in the integration of tidal evolution was introduced by Lagrange and Laplace in the XVIII century. This technique has been widely used to study the stability of the Solar System (e.g., 
Table 1. Astrocentric orbital elements and bulk properties for the configuration of 6 planets in GJ 667C System used in this work. The main values for period, semimajor axe and mass are adopted from Anglada-Escudé et al. (2013). Values for mean, min and max excentricities are results from our model of the dynamical evolution of the system. Radius, in the last column, is estimated by using an interior structure model assuming two extreme compositions: an Earth-like and a Waterworld-like planet. For composition parameters see Table 2

\begin{tabular}{cccccccccc}
\hline Planet & $P(\mathbf{d})$ & $a(\mathbf{A U})$ & $\bar{e}$ & $e_{\min }$ & $e_{\max }$ & $\omega$ & $M_{0}$ & $\boldsymbol{M} \sin i\left(M_{\oplus}\right)$ & $\mathbf{R}\left(R_{\oplus}\right)$ \\
\hline \hline $\mathrm{b}$ & 7.2006 & 0.05043 & 0.08118 & 0.04152 & 0.11711 & 4.97 & 209.18 & 5.94 & $1.6-1.9$ \\
$\mathrm{c}$ & 28.1231 & 0.12507 & 0.02248 & 0.00006 & 0.04806 & 101.38 & 154.86 & 3.86 & $1.4-1.6$ \\
$\mathrm{f}$ & 39.0817 & 0.15575 & 0.04220 & 0.00023 & 0.07449 & 77.73 & 339.39 & 1.94 & $1.2-1.3$ \\
$\mathrm{e}$ & 62.2657 & 0.21246 & 0.02297 & 0.00002 & 0.04809 & 317.43 & 11.32 & 2.68 & $1.3-1.4$ \\
$\mathrm{~d}$ & 92.0926 & 0.27580 & 0.04244 & 0.00016 & 0.08145 & 126.05 & 243.43 & 5.21 & $1.6-1.8$ \\
$\mathrm{~g}$ & 251.519 & 0.53888 & 0.10533 & 0.09696 & 0.11349 & 339.48 & 196.53 & 4.41 & $1.5-1.7$ \\
\hline
\end{tabular}

Laskar 1988). The combination of the orbital forcing with tidal effects was also explored in the specific cases of Venus and Mercury by Correia \& Laskar 2001, 2004 respectively. This method was also applied to exoplanets, first analytically by Wu \& Goldreich (2002), Mardling (2007), Batygin et al. (2009), Laskar et al. (2012), and after by performing numerical simulations in Correia et al. (2010) and Bonfils et al. (2013).

In order to study the detailed evolution of the orbital parameters, we have performed numerical simulations of the system for three possible configurations, spanning a total time of 50,000 years. In all cases, we will assume that the inclination of the planetary system, with respect to us, is $\sim 90^{\circ}$; hence, the observed minimum masses are also the actual planetary masses. In addition, we are using the barycentric coordinates of the system. Figure 1 shows the evolution of eccentricities in one of our simulations for the three configurations. These results show that the dynamic of the system is essentially governed by secular perturbations, i.e. the semi-major axes and eccentricities remain varying between a small range of values throughout all the evolution of the orbits.

However, in the case of 6 planets, 4 of them appear strongly correlated by pairs, $d$ and $f$, and $c$ and $e$. This is evident in the ranges of eccentricities and the average value $\bar{e}$ showed in Table 1 .

The 6-planets configuration, is so dynamically packed that orbital eccentricities oscillate, in some cases, within almost one order of magnitude (see Table 1). Planets $b$ and $g$ are the most eccentric, with upper $e_{b}=0.11711$ and $e_{g}=$ 0.11349 respectively. Planets like $b$ and $d$ perform the largest eccentricity excursions, $e_{b} \approx 0.04152-0.11711$ and $e_{d} \approx$ $0.00016-0.08145$.

In order to introduce the effect of secular perturbations in the tidal evolution equations (see Section 3), we need to estimate the derivative of the eccentricity $(d e / d t)_{\text {pert }}$ at any time for each planetary component. For that purpose, we first calculated the Lomb-Scargle periodogram of the numerical eccentricities for the chosen configurations of the system with: 2, 3 and 6 planets, and identified the periods with the largest power (Lomb 1976, Zechmeister \& Kürster 2009) for the 6-planets configuration. Our results, in this case, are in good agreement with the analytical approach made by Anglada-Escudé et al. 2013.

Using the principal modes, we reconstructed analytically the eccentricity and its derivative. Figure 1 also displays the evolution of eccentricity and its derivative as re- constructed analytically with this procedure for the three configurations.

The analysis of the spectral density, realised by means of Lomb-Scargle's periodograms, indicate that the fluctuations in the eccentricity are periodic and are dominated only by a main mode. On the basis of the parameters in table 6 in Anglada-Escudé et al. (2013), and using the same symplectic integration package (HNBody), and equal parameters for the simulation, we performed the numerical integration of the system to obtain the instantaneous value of the eccentricity of each planet. After a Fourier decomposition, we found an analytical expression that will be used later on the tidal evolution package.

\section{TIDAL EVOLUTION}

Rotational properties of planets and moons (axial tilt and period of rotation) are affected by their tidal interaction with other bodies. In general, these interactions produce a net torque $\mathcal{T}$ that modifies the rotational properties and determines their final equilibrium values. In this work, we assume that the obliquity of the planets in the system is zero. This assumption is not true, because the tilt evolution (tilt erosion) is simultaneous to the spin-orbit evolution. We leave aside this process, but a tilt erosion model is needed for this system.

We calculated the instantaneous rotational rate $\Omega=$ $\dot{\theta}$, if the net torque resulting from the tidal interaction is known, by solving the equation (see Danby 1962):

$$
\frac{d \Omega}{d t}=\frac{\mathcal{T}}{C}
$$

Where $C$ is the moment of inertia around the shortest axis of what will be in general a triaxial body. $C$ can be (in general) written as:

$$
C=\mathcal{C} M_{p} R^{2}
$$

The net torque on a rotating body experiencing tidal interaction with a perturber, has in general two contributions: 1) the triaxial torque that results when the planet has a triaxial shape and, 2) the tidal torque arising directly from the strength on the bulge rose by the perturber. 

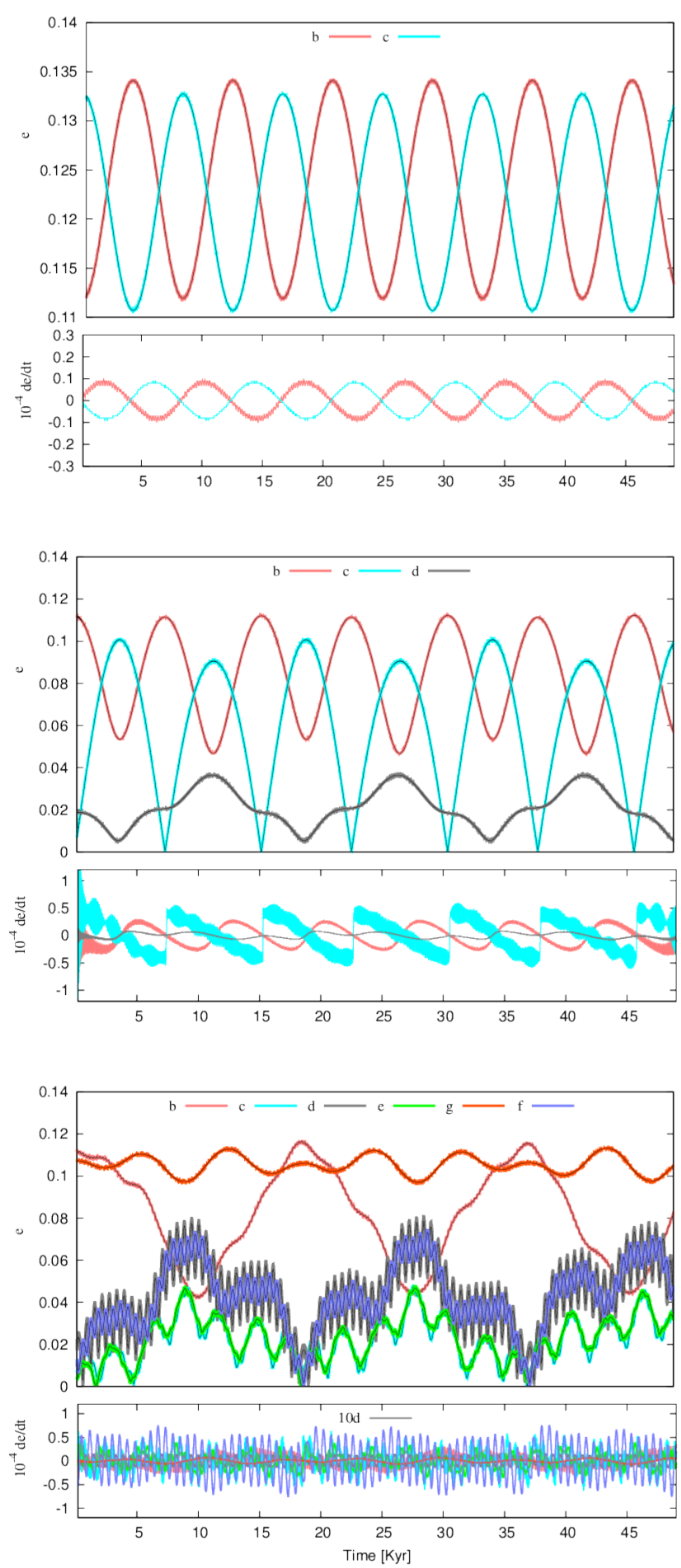

Figure 1. Evolution of eccentricity for GJ 667C system. Three different configurations are showing: 2,3 and 6 planets. The colored curves corresponds to the evolution of eccentricity obtained by numerical integration for each planet. The inner curve in black, inside each colored curve, corresponds to the eccentricity evolution reconstructed semi-analytically for each planet.

\subsection{The Triaxial Torque}

Triaxiality is mainly due to the inhomogeneous distribution of mass in a rotating body, as well as the presence of geological features over the surface. These induce a triaxial shape in the bodies.

Triaxiality is measured in terms of the three principal moments of inertia $A, B$ and $C$ where $A<B<C$ and $C$ is in general given by Eq. (2). The triaxial torque depends on the level of triaxiality as measured by the difference $B-A$ and it is given in terms of the angle between the sideral direction of the bulge $\theta$ and the true anomaly $\nu, \psi=\theta-\nu$. The classic mechanics expression for this torque could be found in Danby (1962) and Goldreich \& Peale (1966),

$$
\begin{aligned}
\mathcal{T}_{\text {tri }} & =\frac{3}{2}(B-A) \frac{G M}{r^{3}} \sin 2 \psi \\
& \approx-\frac{3}{2}(B-A) n^{2} \frac{a^{3}}{r^{3}} \sin 2(\theta-\nu),
\end{aligned}
$$

Here $r$ is the instantaneous distance between the centres of the two bodies.

\subsection{The Tidal Torque}

In the simplest case of a body with radius $R$, interacting with a tide-rising perturber of mass $M$ located at an average distance $a$, the tidal torque is given by the MacDonald (MD) formula (MacDonald 1964, Goldreich 1966, Murray \& Dermott 1999):

$$
\mathcal{T}_{\text {tid }}=\frac{3}{2} G M^{2} \frac{R^{5}}{a^{6}} k_{2} \sin 2\left|\epsilon_{g}\right| \operatorname{Sgn}\left(\epsilon_{g}\right)
$$

where $\epsilon_{g} \equiv(\dot{\nu}-\dot{\theta}) \Delta t$ is the instantaneous geometric lag, i.e. the angle between the direction of the most elongated part of the planet and the line joining the center of the bodies (see figure 4 in Makarov et al. 2012) and $\Delta t$ is a constant time lag. Here $\nu$ is the orbital true anomaly of the perturber and $\theta$ is the sideral angle of the planet measured with respect to the line of apsides.

We observe how the tidal torque strongly depends on the average distance between the bodies $\mathcal{T}_{\text {tid }} \sim \frac{1}{a^{6}}$. For close planets, such as those found around GJ $667 \mathrm{C}$, this effect is proven able to erode any initial rotation period and bring the planet to a low spin-orbit resonance. The timescale for such spin-down from an initial rotation rate $\Omega_{o}$, will be given by the expression from Gladman et al. (1996):

$$
t_{\mathrm{lock}}=\frac{\Omega_{o} C}{\tau_{\mathrm{tid}}}=\frac{2 \Omega_{o} C a^{6} Q}{3 G M^{2} R^{5} k_{2}},
$$

where $Q$ is called the quality factor, which is a specific energy dissipation function. For a rapidly rotating planet $Q \approx 1 / \sin 2\left|\epsilon_{g}\right|$ (Murray \& Dermott 1999).

In a more general case, the tidal torque is obtained from a Fourier expansion of the tide-rising potential that was first developed by Darwin (1879), and more recently updated by Kaula (1964). We called this formalism the Darwin-Kaula (DK) expansion. The Fourier modes, over which this expansion is carried out, are called the tidal modes (Efroimsky \& Makarov 2013), 


$$
\omega_{l m p q} \approx(l-2 p+q) n-m \dot{\theta},
$$

where $l m p q$ are the integers of the Fourier expansion. Here $l$ and $m$ are the degree and order of the expansion respectively, $\dot{\theta}$ is the instantaneous rotation rate of the body and $n$ is the average orbital angular velocity or the mean motion. The DK expansion of the potential provides a rigorous expression for the tidal torque (see equations (109) to (111) in Efroimsky 2012). The resulting expression has two main components: an oscillating component that it is averaged out over an orbit, and a secular component whose average is given by Equation (13) in Makarov \& Efroimsky 2013

$$
\begin{gathered}
\left\langle\mathcal{T}_{\text {tid }}\right\rangle=2 G M^{2} \sum_{l=2}^{\infty} \frac{R^{2 l+1}}{a^{2 l+2}} \sum_{m=0}^{l} \frac{(l-m) !}{(l+m) !} m \times \\
\sum_{p=0}^{l} F_{l m p}^{2}(i) \sum_{q=-\infty}^{\infty} G_{l p q}^{2}(e) k_{l} \sin \epsilon_{l} .
\end{gathered}
$$

Here $F_{l m p}(i)$ and $G_{l p q}(e)$ are known as the inclination and eccentricity functions respectively (Kaula 1961). The most important feature of the DK formula for the tidal torque, in contrast to the MD formula in Eq. (4), is the general dependence on frequency of the love number $k_{l}$, the phase lag $\epsilon_{l}=\omega_{l m p q} \Delta t_{l}\left(\omega_{l m p q}\right)$ and the positive definite time lag $\Delta t_{l}\left(\omega_{l m p q}\right)$. This dependence on frequency is key when treating realistically the rheological properties of planets and moons.

If the bodies are not too close $(R / a<<1)$, eccentricity does not exceed $e \sim 0.3$ and we assume a small obliquity $i \approx 0$, then we can truncate the DK torque in Eq. (7), leaving only the term $l=2$, and $q=-7, \cdot, 7$. This gives us an approximate expression that we will hereafter call the EMW formula,

$$
\begin{aligned}
\left\langle\mathcal{T}_{\text {tid }}\right\rangle & =\frac{3}{2} G M^{2} \frac{R^{5}}{a^{6}} \times \\
& \sum_{q=-7}^{7} G_{20 q}^{2}(e) \mathbf{k}_{\mathbf{2}}\left(\omega_{q}\right) \sin \left|\epsilon_{2}\left(\omega_{q}\right)\right| \operatorname{Sgn}\left(\omega_{q}\right)+ \\
& \mathcal{O}\left(e^{8} \epsilon\right)+\mathcal{O}\left(i^{2} \epsilon\right)
\end{aligned}
$$

Here we have written $\omega_{q}=\omega_{220 q}$. The Kaula functions $G_{20 q}$ are power series of eccentricity related to Hansen's coefficients given by Giacaglia (1976) via

$$
G_{20 q}=X_{2+q}^{-3,2}
$$

In order to calculate the tidal torque using the EMW formula, we first need to obtain the explicit dependence on frequency of the second degree Love number $k_{2}$ and the respective phase lag $\epsilon_{2}$. A rigorous treatment of the problem has been developed in Efroimsky (2012) who studied the response of a viscoelastic near-spherical body to tidal stress. The result of this careful analysis provide us with the value of the $k_{2}$ as a function of planetary bulk properties,

$$
k_{2}=\frac{3}{2} \frac{1}{\left(1+57 \mu / 8 \pi G \rho^{2} R^{2}\right)},
$$

where $\mu$ is the rigidity and $\rho$ the planetary average density. On the other hand, the tidal-mode-dependent phase lag $\epsilon_{2}$ is given by,

$$
k_{2}\left(\omega_{q}\right) \sin \epsilon_{2}\left(\omega_{q}\right)=-\frac{3}{2} \frac{A_{2} \mathcal{I}}{\left(\mathcal{R}+A_{2}\right)^{2}+\mathcal{I}^{2}} \operatorname{Sgn}\left(\omega_{q}\right),
$$

where $\mathcal{R}$ and $\mathcal{I}$ are dimensionless real and imaginary parts of the complex compliance and $A_{2}$ is a coefficient which depends on rigidity, radius and mass of the body (see Apenddix B in Makarov \& Efroimsky 2013):

$$
\begin{gathered}
\mathcal{R}=1+\left(\mathcal{X}_{q} \tau_{A}\right)^{-\alpha} \cos \left(\frac{\alpha \pi}{2}\right) \Gamma(\alpha+1), \\
\mathcal{I}=-\left(\mathcal{X}_{q} \tau_{M}\right)^{-1}-\left(\mathcal{X}_{q} \tau_{A}\right)^{-\alpha} \sin \left(\frac{\alpha \pi}{2}\right) \Gamma(\alpha+1),
\end{gathered}
$$

Here $\mathcal{X}_{q}=\left|\omega_{q}\right| \approx|(2+q) n-2 \dot{\theta}|$ are the so called forcing frequencies. $\alpha$ is a dimensionless parameter (the Andrade's exponent) that depends on the rheological properties of the material that makes up the planet. For materials ranging from ice to most minerals $\alpha=0.14-0.4 . \tau_{A}$ and $\tau_{M}$ are known as the inelastic Andrade's time and the viscoelastic Maxwell's time respectively. These timescales characterise the inelastic and viscoelastic properties of the planetary mantle, and its response to tidal effects in different range of frequencies (Andrade at high frequencies and Maxwell at low frequencies respectively).

Our numerical experiments have shown that the assumption for simplicity $\tau_{A}=\tau_{M}$ does not significantly change the results integrating the tidal evolution, as well as the possibility of being trapped in a given resonance.

\subsection{Tidal Energy Dissipation}

Tidal interaction does not only modify the rotation of the planet. The work done by tidal forces is dissipated as heat inside the deformed bodies and, as a consequence, the orbital energy and angular momentum of the planet change in time. The body acts like a harmonic oscillator dissipating energy during each cycle.

The work done by the drag force, over a displacement during a given interval of time, results in a rotational energy dissipation during each cycle. If the only torque acting on the body is the tidal torque, the thermal energy released as heat is virtually equal to the work done by the tide. Therefore, the dissipated energy will be proportional to the phase lag (Ferraz-Mello et al. 2008) and tide frequency (Efroimsky 2012). Although we have included a triaxial torque, this force do not produce any net dissipated energy. The power dissipated will be simply given by,

$$
\frac{d E}{d t}=-\sum_{q=-7}^{7} \mathcal{T}_{\text {tid }, \mathrm{q}} \omega_{q}
$$

Where $\mathcal{T}_{\text {tid,q }}$ is each of the terms in the sum defining the average tidal torque in Eq. (8). The torque produces a change in the orbital energy of the planet,

$$
E=-\frac{G M_{p} M}{2 a}
$$


Table 2. Notes: (1) Values are different for each planet and appear in Table 1 (2) Values depend on specific numerical experiments.

\begin{tabular}{|c|c|c|c|c|c|}
\hline & Symbol & Units & Earth-like & Waterworld & References \\
\hline \multicolumn{6}{|l|}{$\overline{\text { Planetary Bulk }}$} \\
\hline Planetary Mass & $M_{\mathrm{p}}$ & $\mathrm{kg}$ & (1) & (1) & Anglada-Escudé et al. $(2013)$ \\
\hline Planetary Radius & $R_{\mathrm{p}}$ & $\mathrm{km}$ & (1) & (1) & Zuluaga et al. $(2013)$ \\
\hline Ice mass fraction & IMF & - & $0 \%$ & $50 \%$ & This work \\
\hline Mantle mass fraction & MMF & - & $70 \%$ & $20 \%$ & This work \\
\hline Core mass fraction & CMF & - & $30 \%$ & $30 \%$ & This work \\
\hline Triaxiality & $(B-A) / C$ & - & $5 \times 10^{-5}$ & $<10^{-7}$ & This work \\
\hline \multicolumn{6}{|l|}{ Rheology } \\
\hline Andrade exponent & $\alpha$ & - & 0.3 & 0.14 & Efroimsky \& Lainey $(\overline{2007})$ \\
\hline Bulk Viscosity & $\eta$ & $\mathrm{Pas}$ & $10^{20}$ & $10^{18}$ & Zuluaga et al. $(2013)$ \\
\hline Rigidity & $\mu$ & $\mathrm{Pa}$ & $8 \times 10^{10}$ & $8 \times 10^{9}$ & Moore \& Schubert (2003) \\
\hline Maxwell time & $\tau_{\mathrm{M}} \equiv \eta / \mu$ & $y r$ & 40 & 4 & This work \\
\hline \multicolumn{6}{|l|}{ Star and Orbit } \\
\hline Mass of the star & $M_{\star}$ & $M_{\odot}$ & 0.33 & 0.33 & Anglada-Escudé et al. \\
\hline Initial eccentricity & $e_{o}$ & - & (1) & (1) & Anglada-Escudé et al. $(\overline{2013})$ \\
\hline Initial semi-major axis & $a_{o}$ & $A U$ & (1) & (1) & Anglada-Escudé et al. $(\overline{2013})$ \\
\hline Initial true anomaly/sideral angle & $\theta_{o}$ & - & (2) & (2) & Makarov et al. $(2012)$ \\
\hline
\end{tabular}

and therefore it implies a variation in the semi-major axis given by,

$$
\frac{d a}{d t}=\frac{2 a^{2}}{G M_{p} M} \frac{d E}{d t}
$$

In a similar way the average tidal torque produces a change in the orbital angular momentum of the planet,

$$
\mathcal{L}=M_{p} \sqrt{G M a\left(1-e^{2}\right)},
$$

Differentiating and taking into account that $d \mathcal{L} / d t=$ $-\left(\left\langle\mathcal{T}_{\text {tid }}\right\rangle+\mathcal{T}_{\text {tri }}\right)$ we found an expression for the rate of variation of the eccentricity (see eq. 18).

\section{THE MODEL}

As we have just shown, the rate of rotation and orbital properties of a planet are modified by the tidal interaction with its host star. The intensity of this interaction, quantified by the tidal and triaxial torques, depends on a set of bulk, interior structure and rheological properties of the planet. The mass of the central star and the initial orbital parameters are also required. In Table 2 we summarize the set of input parameters that are necessary to estimate the net torque on the planets in the GJ $667 \mathrm{C}$ system.

Planetary composition and water cycle models suggest that planets in GJ 667C could be volatile-rich bodies (Schaefer \& Sasselov 2015). In order to study the dependence of the spin-orbit evolution on composition, we assumed two different sets of input parameters (columns 4 and 5 in Table 2). The first set (column 4), hereafter the reference model, corresponds to planets with an Earth-like (El) composition, i.e. composed by a silicate mantle and a metallic core with almost no water content. The second set of parameters (column 5) are chosen to match the bulk and rheological properties of planets containing a significant fraction of water, i.e. Waterworlds (Ww).

Here, we are using the idea of Ocean planets, as was proposed originally by Kuchner (2003) and described carefully later on by Léger et al. (2004) and Adams et al. (2008).
This description considers planets with more than $25 \%$ of mass made by water. The internal structure of this kind of planets is not well known, but the models propose solid cores made by iron-rock, deep layers made by different water ice phases, and massive liquid water oceans.

As the bulk structural properties of this $\mathrm{Ww}$ are unknown, we consider that this iron-rocky-ices-water (solidliquid) planets must have an internal structure similar to Ganymede (Vance et al. 2014). Makarov (2015) called this kind of bodies semiliquid, planets with massive surface oceans and rigid cores that possess a very low but nonzero triaxiality. In our case, we ran numerical experiments using a low triaxiality torque for $\mathrm{Ww}$.

To follow the spin-orbit evolution, we need to solve the following set of coupled, first order, non-linear differential equations:

$$
\begin{aligned}
\frac{d \theta}{d t} & =\Omega \\
\frac{d \Omega}{d t} & =\left[\left\langle\mathcal{T}_{\text {tid }}\right\rangle(a, e, \Omega)+\mathcal{T}_{\text {tri }}(a, e, \theta, t)\right] / C \\
\frac{d a}{d t} & =-\frac{2 a^{2}}{G M_{p} M} \sum_{q=-7}^{7} \mathcal{T}_{\text {tid }, \mathrm{q}}(a, e, \Omega) \omega_{q} \\
\frac{d e}{d t} & =\left(\left\langle\mathcal{T}_{\text {tid }}\right\rangle+\mathcal{T}_{\text {tri }}\right)\left(\frac{a\left(1-e^{2}\right)}{G M}\right)^{1 / 2} \frac{1}{e a M_{p}}+\frac{\dot{a}}{2 e a}\left(1-e^{2}\right)
\end{aligned}
$$

It should be noted that these equations only take into consideration the tides on the planet. The tides risen by the planet on the star are neglected.

With initial conditions $\theta(0)=\theta_{o}, \Omega(0)=\Omega_{o}, a(0)=a_{o}$ and $e(0)=e_{o}$. The previous set of equations do not take into account the effect that secular perturbations of other planets in the system have on the spin-orbit evolution.

\subsection{Numerical Experiments Using TIDEV}

In all our numerical experiments on the spin-orbit evolution, we use a script written in $\mathrm{C}++$ language that we named TIDEV. The script is available for public users in https://github.com/facom/tidev. 
TIDEV is a general framework developed to calculate the evolution of rotation for tidally interacting bodies using the formalism proposed by EMW. Our numerical tool performs the integration of the set of equations (18). It computes the rotational and dynamical evolution of a planet under tidal and triaxial torques. TIDEV also takes into account the perturbative effects due to the presence of the other planets in the system, especially the secular variations of the eccentricity.

The input parameters are divided in those related with the bulk composition and rheology of the planet and those related with the initial orbital configuration of the system.

Typical bulk parameters are the mass and radius of the planet (and those of the other planets involved in the integration), the size and mass of the host star, the Maxwell time and Andrade's parameter (see Table2).

To give the initial orbital configuration, we need to have an initial set of orbital elements (see Table 1). The two initial conditions needed for each integration are the sidereal angle of the tidal bulge $(\theta)$ and the initial rate of rotation $\Omega$. This later value is given as a function of the mean motion $n$ and depends on the resonance under study; for example, if we need to run a simulation in the vicinity of $3: 2$ resonance, the initial rotational velocity reads $\dot{\theta}_{0}=1.51 n$.

For the computation of the locking time, we have adopted the assumption that the initial period of rotation is 24 hours. Then, the value of $\Omega_{0}$ for this case is just $\Omega_{0}=(2 \pi / 86400) s^{-1}$.

For our purposes, the most important value returned by TIDEV is the time evolution of the rate of rotation of the planet, which enables us to estimate the capture probabilities in each resonance considered in this work.

As usual in any scheme of numerical integration, determining a sufficiently good time step is crucial for the success of the integration. With a gross value of the time step (for example, if it is comparable with the orbital period), we can lose important information about the rotation history of the planet. On the other hand, a short time step increases dramatically the computation time and also the machine precision becomes a concern. As a general rule in our integrations, we need to use a time-step not greater than a fraction of a terrestrial day. In spite of this, even with this selection of the time step, the integrations are expensive in a computational sense.

A new numerical tool was developed and presented by Bolmont et al. (2015). Their model is based on the classic programme Mercury for N-bodies, and has been extended to include the effects of the tidal torque, the deforming effect of the rotation and the relativistic effects produced by the nearness of the planet to its star.

The tidal model used by Bolmont et al. (2015) is the classic model of constant lag by Hut (1981). Though the model does not define explicitly the tidal torque, it is expressed in terms of momentum (see equation (5) in Bolmont et al. (2015)). Unlike our model, theirs lack of the possible effects that would have a change in the bulk composition of the planets, and therefore, their model does not include changes in the rheology. Also, it does not include calculations of the possible effects caused by other planetary members in the system, this is, additional gravitational disturbances.

On the other hand, their model calculates, like ours, the time scale that a planet takes to be tidally locked as well as the periods of rotation reached at the end of the spin-orbit evolution.

The main disadvantage in the work of Bolmont et al. (2015) is the frequency-independent tidal model. Their approach introduce the rotational evolution separated from the tidal forces, the deformation and the relativistic effect, which is not ideal from our point of view. In our case, the calculation of gravitational interactions between planets was done previously, then we included in the rotational evolution integration.

In order to make a simple quantitative comparisson between Bolmont's model and our model, we made the same computations for Kepler-62 b with the parameters given in table (7) of Bolmont et al. (2015) for the semimajor axis and eccentricity. Regarding the bulk properties of the planet, we assume a mass $5 \%$ higher than the nominal value as explained in their work (i.e. equal to $2.72 M_{\oplus}$ ) and a radius of $1.31 R_{\oplus}$. The rheological parameters were taken equal to those of an El planet, listed in Table 2 of this work. We computed the capture probabilities around $3: 2$ and $1: 1$ resonances and obtained a capture probability of $41.45 \%$ in $3: 2$ resonances yielding a $58.55 \%$ probability of being captured in the synchronous resonance. These results support the idea (only for planet Kepler-62 b) presented in Bolmont's work that the three inner planets of the systems are slow rotators with rotation periods grater than $100 \mathrm{hrs}$. Similarly, our results for the locking time are in the same order of that from Bolmont $\left(10^{3} \mathrm{yr}\right)$. We need to clarify that in these simulations we did not take into account the evolution of the obliquity, only that of the rotational period.

Turning to our model, there are two interesting quantities to compute for each planet: (1) the spin-down time, $t_{\text {lock }}$, i.e. the time required for an almost complete erosion of any initial rotation rate $\Omega_{0}$ until it reaches a resonant state where the rotational angular velocity is close to the orbital angular velocity; $(2)$ the capture probability $P_{c}$, i.e. the fraction of times the planet is trapped in a given spin-orbit isolated resonance where rotation and orbital angular velocities become commensurable, $\Omega / n \approx(2+q) / 2$ with $q=0,1,2 \cdot$. As a side note, the $1 / 2$ spin-orbit resonance $(q=-1)$ is also possible under certain conditions, if the planet evolves from an initial retrograde spin.

We calculate the spin-down times integrating the set of Equations 18 in a coarse grained time grid. We have verified that semi-major axis and eccentricities are not significantly modified by tidal interaction in this particular system. Therefore, only the first two equations should be solved. If we assume a relatively large value for $\Omega_{o}$, i.e. $\Omega_{o}=7.3 \times 10^{-5} s^{-1}$, the tidal and triaxial torques are small and hence, we can assume, for a given range of time, an average constant tidal torque.

Table 3 shows the spin-down time for a 6 planets configuration and its dependence on the set of planetary properties we assumed ( $\mathrm{El}$ or $\mathrm{Ww}$ planets). For each planet we have also calculated the $t_{\text {lock }}$ using the classical MacDonald's parameters (see Equation 5 ). 

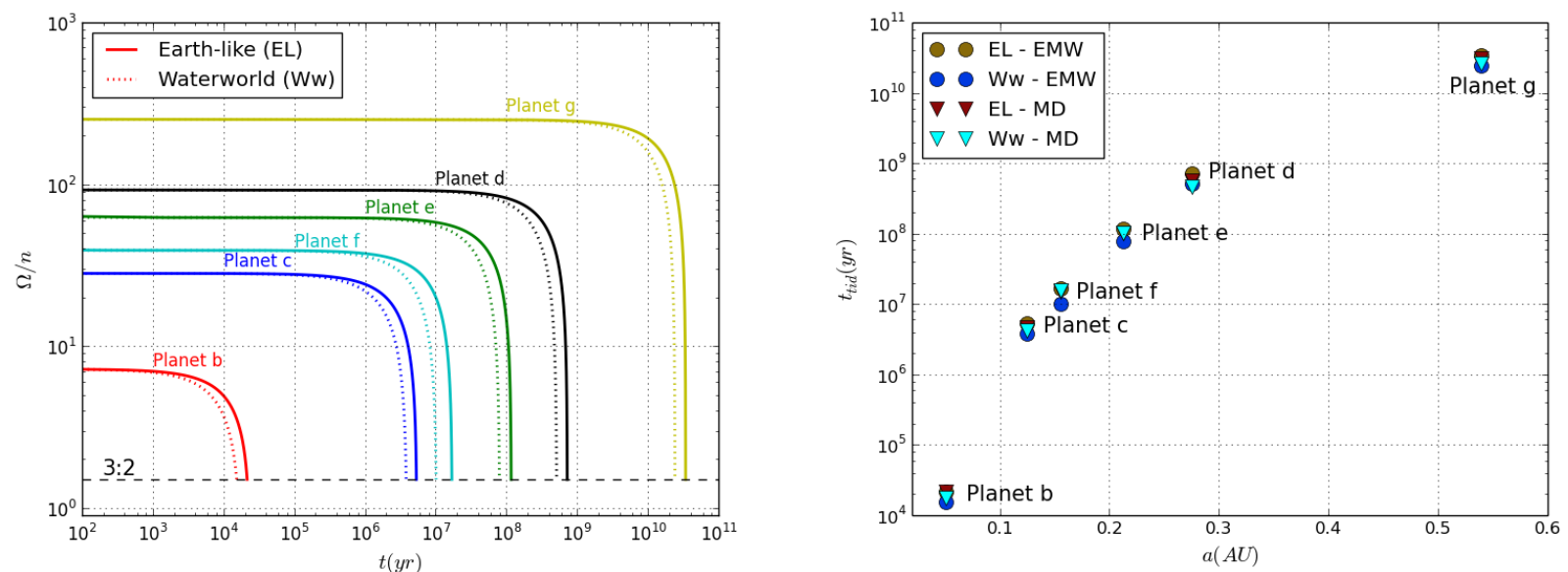

Figure 2. Left panel: spin down times for $\mathrm{El}$ (continuos line) and Ww (doted line) planets. Right panel: Spin-down time comparison between the numerical integration using EMW and MD models.
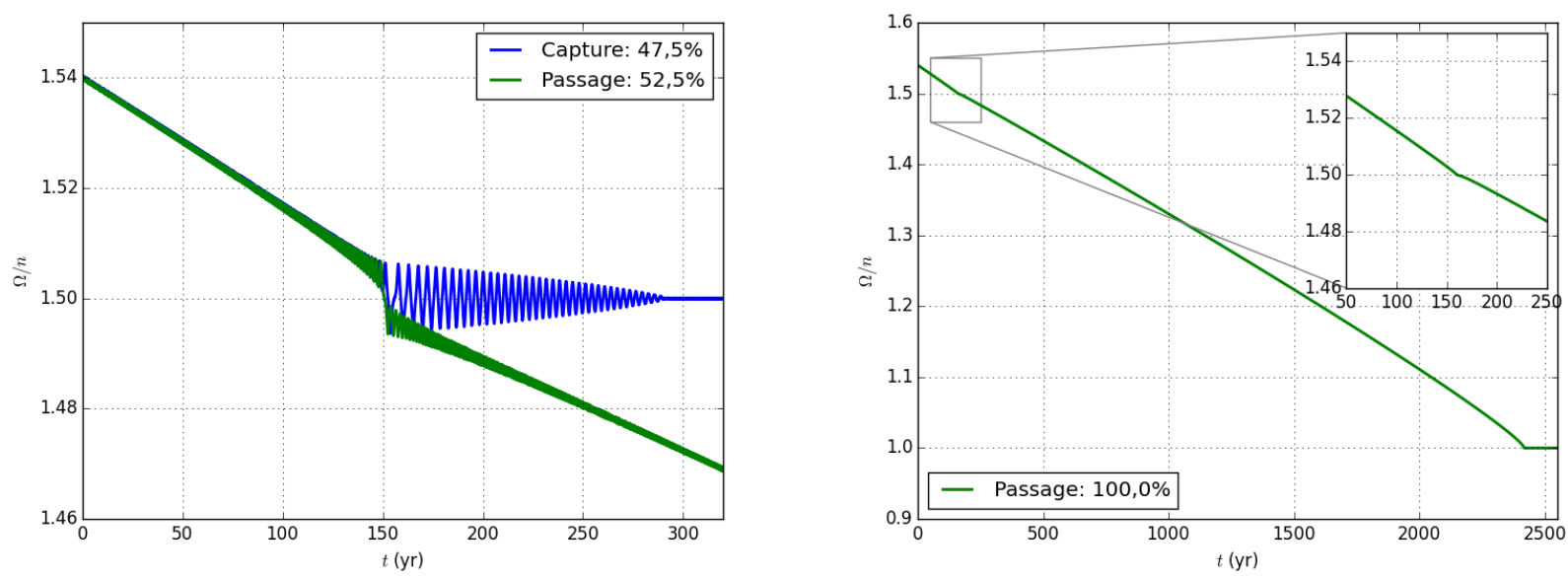

Figure 3. Probabilities to reach 3:2 resonance for Planet $b$, Earth-like (left panel) and Waterworld (right panel).

\section{RESULTS}

Our main results are related with the probability to reach low resonances and its dependence on composition, changes on eccentricity and interactions with the other bodies in the system.

First, we analyse the influence of the secular changes in the orbital elements, i.e. changes in eccentricity and semimajor axes (see Section 2). On the other hand, we developed numerical experiments to determine the dependence of the model on fictitious eccentricity changes.

\subsection{Capture Probabilities}

It is known that the probability of capture depends on the eccentricity and other quantities as the quality factor. We have calculated the capture probability of different spinorbit resonances. For that purpose we use the brute force method used previously by Correia \& Laskar (2004) and Makarov et al. (2012), that for the sake of completeness we will explain here. We start with an initial condition $\Omega(0)$ close to the studied isolated resonance, i.e. $\Omega(0) / n=(2+$ $\eta) / 2+\epsilon$.

Around the isolated resonance, the tidal torque becomes minimum and, as a consequence, the system tends to preserve this idealised state. However, as the planet naturally crosses the resonance, its angular velocity starts to oscillate around the resonant frequency (librations). According to the initial value assumed for $\theta$, the sideral angle formed between the bulge axis and the star, the chances the planet has to traverse this resonance and emerge without being trapped is different.

This is exactly what makes this process stochastic in nature. The number of times the planet is trapped in a given resonance defines the probability of that resonance. We did 40 runs for each initial sidereal angle, for each planet and for two different compositions each time. The same was made to include the interactions with other planets for two different configurations of the system: 3 and 6 planets.

In Table 4, we show the results of the calculated proba- 

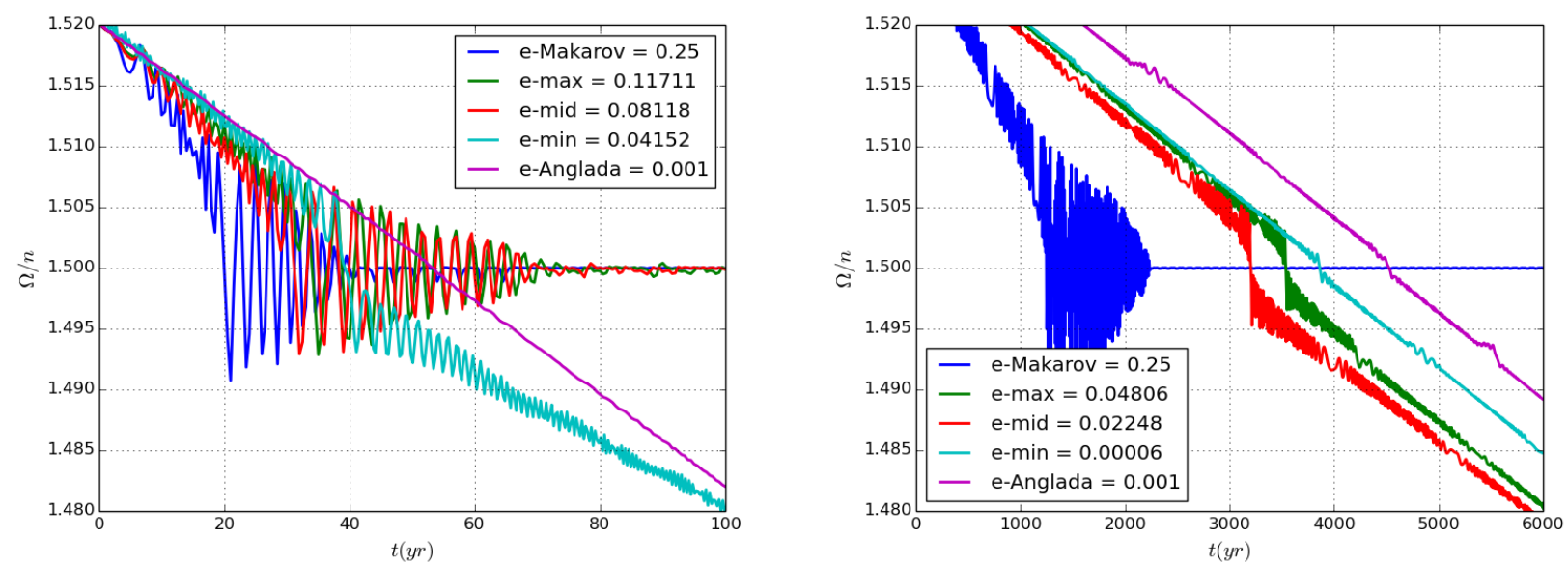

Figure 4. Earth-like planets $b$ (left) and $c$ (right) arriving and passing 3:2 resonance for different eccentricities.

Table 3. Spin-down times in years for all planets in the GJ $667 \mathrm{C}$ system. Times were calculated using both, EMW and MD methods. For EMW calculations, we computed the value of the Love number $k_{2}$ using eq. 10 and calculated an effective $Q_{e}$ for each $t_{\text {lock}}$. For MD calculations, we used $k_{2}$ and $Q$ values from literature: Henning et al. 2009 for Earth-like planets and Barnes et al. 2012 for Waterworlds.

\begin{tabular}{cccc}
\hline & $t_{\text {lock }}(\mathrm{yr})$ & $k_{2}$ & $Q_{e}$ \\
\hline \hline Earth-like - EMW & & & \\
\hline b & $2.16 \times 10^{4}$ & 1.05 & 173.55 \\
$\mathbf{c}$ & $5.34 \times 10^{6}$ & 0.92 & 174.47 \\
$\mathbf{f}$ & $1.69 \times 10^{7}$ & 0.68 & 125.47 \\
$\mathbf{e}$ & $1.16 \times 10^{8}$ & 0.79 & 146.25 \\
$\mathbf{d}$ & $7.25 \times 10^{8}$ & 1.01 & 214.90 \\
$\mathbf{g}$ & $3.42 \times 10^{10}$ & 0.96 & 178.33 \\
\hline Earth-like - MD & & & \\
\hline $\mathbf{b}$ & $2.17 \times 10^{4}$ & 0.3 & 50 \\
$\mathbf{c}$ & $4.67 \times 10^{6}$ & 0.3 & 50 \\
$\mathbf{f}$ & $1.53 \times 10^{7}$ & 0.3 & 50 \\
$\mathbf{e}$ & $1.04 \times 10^{8}$ & 0.3 & 50 \\
$\mathbf{d}$ & $5.68 \times 10^{8}$ & 0.3 & 50 \\
$\mathbf{g}$ & $3.06 \times 10^{10}$ & 0.3 & 50 \\
\hline Waterworld - EMW & & & \\
\hline $\mathbf{b}$ & $1.53 \times 10^{4}$ & 1.39 & 343.55 \\
$\mathbf{c}$ & $3.82 \times 10^{6}$ & 1.34 & 243.35 \\
$\mathbf{f}$ & $1.01 \times 10^{7}$ & 1.31 & 166.67 \\
$\mathbf{e}$ & $7.96 \times 10^{7}$ & 1.34 & 211.06 \\
$\mathbf{d}$ & $5.12 \times 10^{8}$ & 1.39 & 301.23 \\
$\mathbf{g}$ & $2.42 \times 10^{10}$ & 1.37 & 251.96 \\
\hline Waterworld - MD & & & \\
\hline b & $1.75 \times 10^{4}$ & 0.5 & 100 \\
$\mathbf{c}$ & $4.15 \times 10^{6}$ & 0.5 & 100 \\
$\mathbf{f}$ & $1.58 \times 10^{7}$ & 0.5 & 100 \\
$\mathbf{e}$ & $1.01 \times 10^{8}$ & 0.5 & 100 \\
$\mathbf{d}$ & $4.72 \times 10^{8}$ & 0.5 & 100 \\
$\mathbf{g}$ & $2.64 \times 10^{10}$ & 0.5 & 100 \\
\hline & & &
\end{tabular}

bilities of capture in three isolated resonances $(5: 2,2: 1,3: 2)$, for planets $b$ and $c$. The table shows the results of including the tidal interactions between the planet and the host star and the gravitational interaction of that planet with other bodies in the system, in this case, including a configuration of 3 and 6 planets.
Table 4. Probability of capture for planets b and c, for Earthlike (El) and Waterworld (Ww) composition for three different configurations: 1) Star-planet, 2) Star-planet +2 more planets (including perturbations), and 3) Star-planet +5 more planets. The eccentricity used for El planets is $\bar{e}$ (see Table 1).

\begin{tabular}{lcccc}
\hline & $\mathrm{e}$ & $3: 2$ & $2: 1$ & $5: 2$ \\
\hline \multicolumn{5}{c}{ Star - planet } \\
\hline \hline El b & 0,08 & $47,5 \%$ & $10,2 \%$ & $0,0 \%$ \\
El c & 0,02 & $10,0 \%$ & $0,0 \%$ & $0,0 \%$ \\
\hline Ww b & 0,08 & $0,0 \%$ & $0,0 \%$ & $0,0 \%$ \\
Ww c & 0,02 & $0,0 \%$ & $0,0 \%$ & $0,0 \%$ \\
\hline \multicolumn{5}{c}{ Star - 3 planets } \\
\hline \hline El b & 0,08 & $41,0 \%$ & $15,0 \%$ & $0,0 \%$ \\
El c & 0,02 & $0,0 \%$ & $0,0 \%$ & $0,0 \%$ \\
\hline \multicolumn{5}{c}{ Star - 6 planets } \\
\hline \hline El b & 0,08 & $41,0 \%$ & $15,4 \%$ & $0,0 \%$ \\
El c & 0,02 & $0,0 \%$ & $0,0 \%$ & $0,0 \%$ \\
\hline \hline
\end{tabular}

For planet $b$ (the closest planet), the dependence of resonance capture on bulk composition is clear, when you change from El to $\mathrm{Ww}$ composition, the probability of capture down to zero for any resonance higher than 1:1. For an El composition, this planet reaches the 3:2 resonance in $47.5 \%$ of the cases in a period of time less than $22 \mathrm{Kyr}$.

When other planets are included, the probability varies from $47.5 \%$ to only $41 \%$ for resonance $3: 2$, but for resonance $2: 1$ the probability increases from $10.2 \%$ to $15.0 \%$. These changes are caused by the periodic variations in eccentricity when other bodies are added to the system.

In the case of planet $c$, for El composition the planet ends $10.0 \%$ of the cases in a resonance $3: 2$, in a time less than 5.5 million years.

For the $\mathrm{Ww}$ case, and for both planets, $b$ and $c$, the probability of capture in any resonance is $0.0 \%$ for low eccentricities.

Considering that the actual composition of the planets remains unknown, these numerical experiments just address us to an idea about the behaviour of the real planets. However, the rheology of the planet provides us with information about the tendency of planets with different viscoelastic be- 


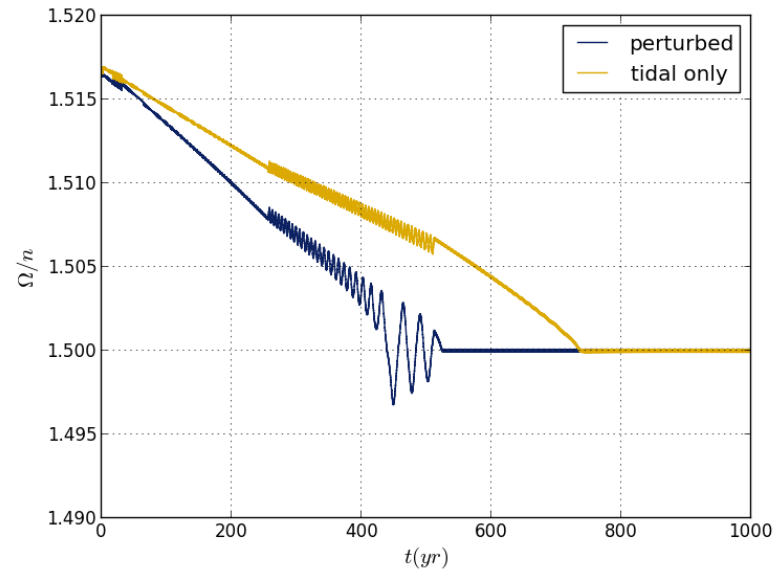

Figure 5. El planet $c$ perturbed gravitationally by all the other members of the system (6 planets configuration) compared with a non perturbed (tidal only) Star-planet configuration.

haviour to becoming trapped in a specific resonance. At first sight, planets with low average density tend to cross resonances like $2: 1$ or $3: 2$ and get the $1: 1$ resonance directly.

On the other hand, although the triaxiality of the body produces a measurable torque, after analysing the dynamic evolution of the system and obtaining the calculations of probability for a 3:2 resonance with the EL planet $c$, we can assure that the contribution of the triaxial torque to the secular evolution of eccentricity, is negligible. In Figure 7 we can see planet $c$ arriving to the resonance $3: 2$ in both cases, with and without considering the triaxial torque. The probabilities of capture are not modified if we includes the triaxial torque.

In the case of Waterworlds, we developed numerical experiments within a range of low triaxialities $\left(<10^{-7}\right)$, even zero in some cases, and we found that for this kind of planets the probability of capture in any resonace higher than 1:1 is zero for low eccentricities (see table 4).

When you have low values of the triaxiality, capture in a given resonance is achieved for smaller values of the eccentricity (see fig 8 in Makarov 2012). In other words, some resonance of low order is more likely to occur if the traxiality tends to zero, which has been verified with our calculations.

\subsection{Changes on the Eccentricity}

The effects of the eccentricity variation produced by the gravitational interaction between the planets have been introduced in the rotational evolution, entered through an analytical expression obtained from a Fourier transformation of the eccentricities, obtained in turn through a numerical integration (see Section 2). It is important to note that the time in which the eccentricities evolve is much larger than the orbital and rotation rates of the planets.

Therefore, to evaluate the impact of the eccentricity on the capture probabilities, first we took three representative values of eccentricity for each planet: $e_{\min }, \bar{e}, e_{\max }$ (see Table 1), and studied the evolution of the rotational rate of various spin-orbit resonances. We found that in any

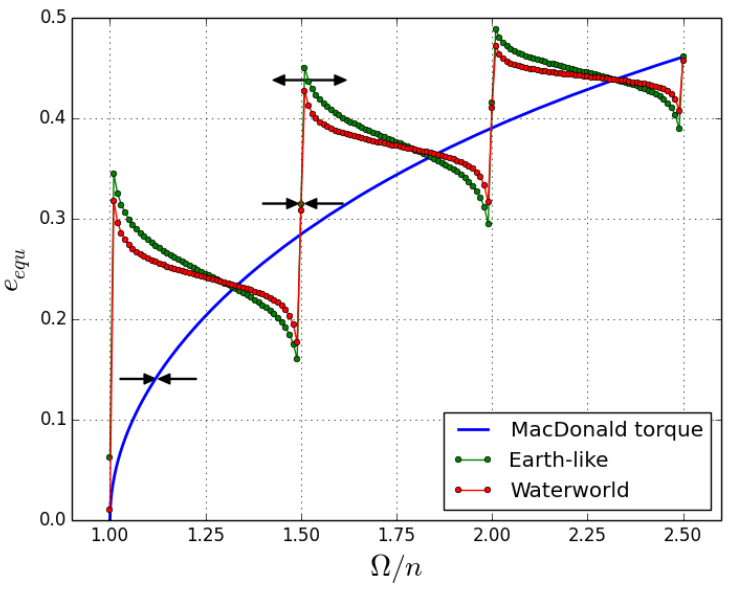

Figure 6. Resonance depending on an equilibrium eccentricity for both compositions in the case of planet $c$.

case the final captured probabilities were not altered. We include calculations made with other values of the eccentricity obtained by Anglada-Escudé et al. (2013) and Makarov \& Berghea (2014) also (see Figure 4).

A particularly interesting dynamical scenario arises when mean tidal torque vanishes. This situation leads to a state of dynamical equilibrium where the disturbed body spins with constant angular velocity (Makarov 2012).

Depending on the form of the torque, we can infer the values of eccentricity for which it vanishes giving a fixed value of $\dot{\theta}$. The first model is the Constant Time Lag (MD model) which sets the values of the time delay $\Delta t$ as independent of the tidal mode frequency. In this model, the mean tidal torque involves the following terms (Hut 1981; Makarov \& Efroimsky 2013):

$$
<\tau_{\text {tid }}>\propto\left[\frac{1+\frac{5}{2} e^{2}+\frac{45}{8} e^{4}+\frac{5}{16} e^{6}}{\left(1-e^{2}\right)^{6}}-\frac{\dot{\theta}}{n} \frac{1+3 e^{2}+\frac{3}{8} e^{4}}{\left(1-e^{2}\right)^{9 / 2}}\right]
$$

By setting $\left\langle\tau_{t i d}\right\rangle=0$ we obtained the following approximate expression for the angular velocity as a function of the eccentricity and the mean motion taken as a constant

$$
\dot{\theta}=n\left[1+6 e^{2}+\frac{3}{8} e^{4}+\frac{173}{8} e^{6}+\mathcal{O}\left(e^{8}\right)\right]
$$

which can be solved for the eccentricity for a given resonant or non-resonant value of $\dot{\theta} / n$. This root is called the equilibrium eccentricity. The behaviour of this eccentricity is depicted by the blue rising curve in Figure 6. The region above the blue line corresponds to positive values of the mean torque; therefore, in this zone the torque accelerates the rotational motion of the body. Below the blue line, the torque is negative and it contributes to despin the body. In this figure we can see the intrinsically stable behaviour of the equilibrium states in the sense that any horizontal perturbation made on a point of the curve (increasing or decreasing the rotational velocity) is counteracted by a torque which tends to return the motion to the original state as depicted by the direction of the black arrows. 

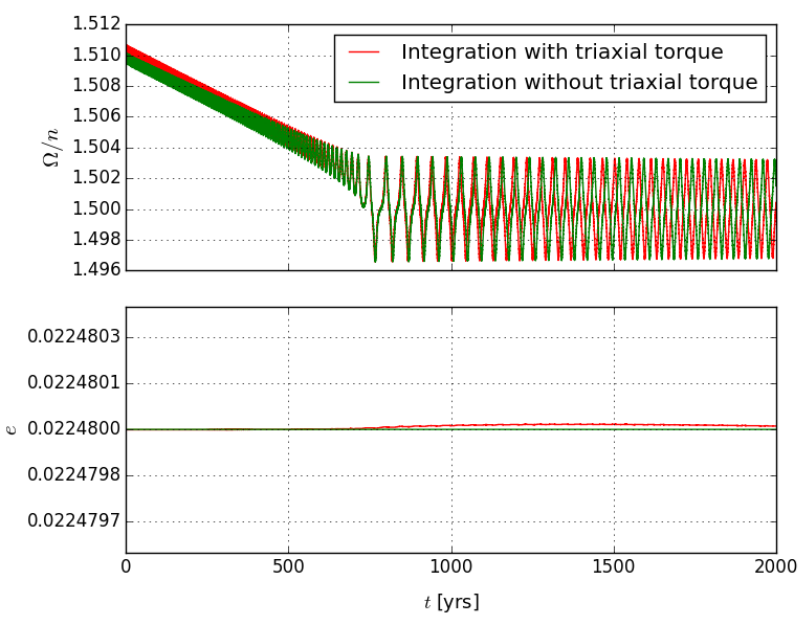

Figure 7. Earth-like planet $c$ eccentricity evolution, including both, tidal and triaxial torques.

We proceeded in the same way with the EMW form of the tidal torque in order to explore the concept of equilibrium eccentricity in this model. We solved Equation (8) for the eccentricity contained inside the $G_{20 q}$ functions for a set of resonant and non-resonant values of $\dot{\theta} / n$ ranging from 1:1 to $5: 2$. As we can see from the red and green curves in Figure 6, the behaviour of equilibrium eccentricity is completely different in this case. Perturbing any arbitrary but non-resonant point of the curve, leads to torques which act in the same direction of the perturbation in contrast to the MD case. Therefore, the equilibrium of points outside any resonance is unstable by nature in this formalism. On the other hand, when we disturb a resonant point (the central point along the nearly vertical lines), two torques emerge in opposite direction to the perturbation counteracting its effect. From this discussion, in the MD model any possible $\dot{\theta} / n$ represents a state of stable equilibrium, but with the EMW torque, only resonant values are stable, if are coupled with the appropriate value of eccentricity. In both, the MD and in the EMW torque, we only have one eccentricity value for which the average torque is zero, but this does not mean that it is stable in the last case (see Section 3 in Makarov \& Efroimsky (2013)).

In an empirical sense, we can make general conclusions about the final spin state of a body from the equilibrium eccentricity in Figure 6 In fact, if we introduce a fictitious perturbation that covers a range of eccentricities wide enough to contain one of the resonant and stable values of the angular velocity, the probability of capture in that resonance is no longer zero no matter how high the resonance is. We made an experiment with planet $c$ introducing a fictitious sinusoidal perturbation with an amplitude of 0.4 . We found that the most probable resonance is $3: 2$ with a probability of $85 \%$. But interestingly, the next most probable resonance was $2: 1$ with the remaining $15 \%$ of the probability. This experiment confirms our suspicion that providing a wide perturbation to the eccentricity, there could be capture in high order resonances. This idea was discussed previously in classic works as Goldreich \& Peale (1966).

\subsection{Semi-analytical Probability}

Another semi-analytical method to get a probability of capture, is considering the librations around the resonance as proposed by Goldreich (1966) and Goldreich \& Peale (1968). In this method, the capture depends on the change of the kinetic energy at the end of the libration and its relation with the total energy dissipation in a complete cycle.

The semi-analytical formula for the probability derived by Goldreich (1966) adapted to the DK torque gives:

$$
P_{\text {capt }}=\frac{2}{1+2 \pi V / \int_{-\pi}^{\pi} W(\dot{\gamma}) d \gamma}
$$

where

$V=K \sum_{q \neq q^{\prime}} G_{220 q}^{2} k_{2}\left(\left(q-q^{\prime}\right) n\right) \sin \left|\epsilon_{2}\left(\left(q-q^{\prime}\right) n\right)\right| \operatorname{Sgn}\left(q-q^{\prime}\right)$

and

$$
W(\dot{\gamma})=-K G_{220 q^{\prime}}^{2} k_{2}(\dot{\gamma}) \sin \left|\epsilon_{2}(\dot{\gamma})\right| \operatorname{Sgn}(\dot{\gamma})
$$

being $K$ a positive constant which does not play a role in the computation of capture probability. Numerical integration of this equation for different values of eccentricity gives us insight on the strong dependence of both quantities. In fact, for planet $b$, we found that the capture in $3: 2$ resonance is guaranteed for eccentricities equal or greater than 0.15 . On the other hand, the probability of capture in $2: 1$ is almost $23 \%$ for that same eccentricity $(e=0.15)$, then the capture in this resonance is also possible (see Figure 9).

To evaluate this integral, we must consider the following separatrix equation which relates the parameters $\dot{\gamma}$ and $\gamma$, which define the phase space trajectory near a given resonance.

$$
\dot{\gamma}=2 n\left[3 \frac{(B-A)}{C} G_{20 q^{\prime}}(e)\right]^{1 / 2} \cos \frac{\gamma}{2}
$$

We performed a series of experiments with El planets $b$ and $c$ to compare both methods: the brute force and the semi-analytical one. For planet $b$, with the former method, we obtained a capture probability of $47.5 \%$ in the $3: 2$ resonance and, with the semi-analytical method, we obtained for the same planet and resonance a probability of $\sim 50.0 \%$, which represents a reasonably good agreement between both methods (see Figure 9). The same comparison was performed with planet $c$ and we got $10.0 \%$ and $\sim 6.0 \%$ of capture probability in 3:2 resonance with the two methods respectively.

This semi-analytical method was also applied to the Ww versions of planets $b$ and $c$, but the results in this case were quite different from those obtained by brute force. However, we do not believe the semi-analytical formula can be used in this case because it depends strongly on the behaviour of $(B-A) / C$. In fact, the separatrix equation only takes into account the triaxial torque, but not the tidal torque. The effects of the last one are estimated by the energy dissipation calculated using the phase space trajectory which is determined by $(B-A) / C$. Since $\mathrm{Ww}$ planets do not have a permanent triaxiality, equations 21) and 


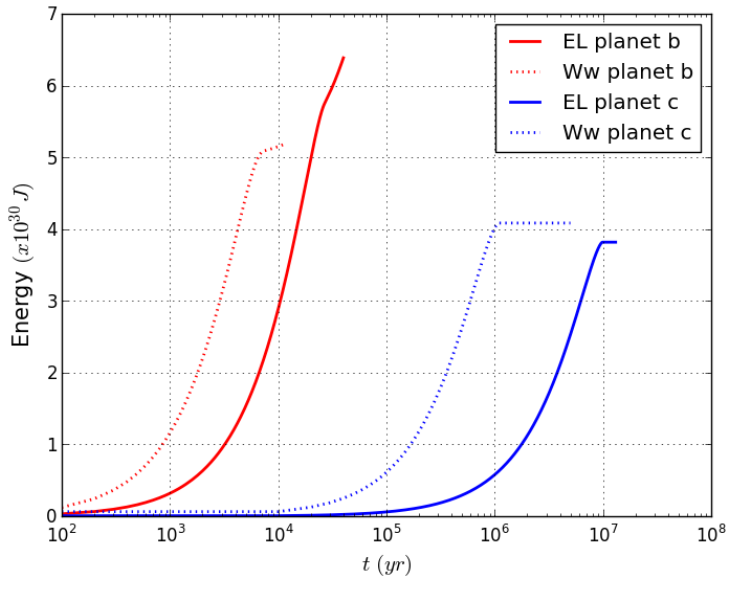

Figure 8. Tidal energy dissipated by El and $\mathrm{Ww}$ planets $b$ and c.

24) are inappropriate for computation of the isolated resonance capture probability (Makarov, personal communication). Then, the comparison between two methods carried out in this work is limited to El planets.

\subsection{Energy Dissipation}

We used the results for the tidal energy dissipation to compare the times of locking for the two different compositions. In Figure 8 we show how El planets take more time to dissipate the tidal energy and finally reach the resonance.

But according to the classical expression (see eq. (5)), this time is directly proportional to the quality factor $Q$. Why do $\mathrm{Ww}$, that have a greater $Q$, take less time to reach the resonance? We deduced that in this case the size of the planet has more effect over the spin-down time than the quality factor.

As we can see in Table 3 the effective quality factor for $\mathrm{Ww}$ are 2 times greater than the El planets. The spindown times for these planets should also be 2 times greater. On the other hand, $t_{\text {lock }} \sim R^{-5}$, according to this, bigger planets take less time to reach a final low resonance. They dissipate tidal energy rapidly compared to small planets, even when having a greater $Q$.

As a result, the dissipation of tidal energy is related to the planetary composition and its rheology. According to our results, water-rich planets achieve the low resonance or the total tidally locked state, far less than rocky planets.

\section{DISCUSSION AND CONCLUSIONS}

We have produced an updated dynamical study of the GJ667C planetary system. We have verified that the spinorbit evolution of the planets is exclusively secular. This result agrees with that of Anglada-Escudé et al. (2013). In our tidal model, we also computed the energy dissipated by tidal friction and the possible associated variation of the orbital elements. However, we found that the semi-major axis and eccentricities are not significantly modified by tidal interactions in this particular system.

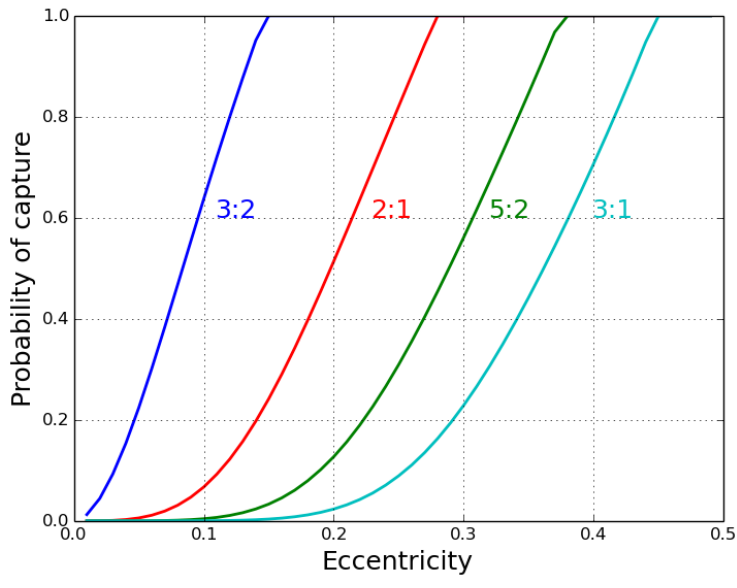

Figure 9. Probability of an isolated resonance capture depending on the eccentricity for an El planet $b$. We use the expression of probability by Goldreich (1966).

We have calculated the spin-down times until it reaches a low resonance for a 6-planet configuration. For this specific configuration and for the reported age of the syetem, we found that the equilibrium spin-rotation has probably been achieved for planets $b, c, e$ and $f$ in a low resonance. Planet $g$ is probably rotating close to its original rate, in the case of planet $d$ it may not have achieved any final spin-orbit resonance, this depending on the actual age of the system.

We calculated the capture probabilities of isolated resonances using three different configurations of gravitational perturbations in the planetary system: star-planet, starthree planets and star-six planets. In agreement to the work by Anglada-Escudé et al. (2013), we used a 6 compact planetary system. On the other hand, and taking into account the work by Feroz \& Hobson (2014), we used two other configurations including 2 and 3 planets.

\subsection{Perturbations of Others Planets}

We computed the secular evolution of the system with 2, 3 and 6 planet configurations. If we consider the probable age of the system $\sim 8 \mathrm{Gyr}$, the absence of a substantial variation on the semi-major axis is a clear evidence of the system stability.

The eccentricities of the planets suffer mainly secular variations. Planets $b$ and $d$ experience the largest eccentricity changes (see Section 2). In the case of a 6-planets configuration, some of them show strong correlations by pairs, $d$ and $f$, and $c$ and $e$. The presence of other planets produces an eccentricity variation around an average value (see Table 1) that results in a main frecuency for all the planets around $17000 \mathrm{yr}$.

We calculated the probability of resonance for El planets $b$ and $c$ including the perturbations of the other planets. We included planet $d$ in a 3-planets configuration, and $e$, $f$ and $g$ in a 6-planets configuration. The results, shown in Table 4 let us conclude that the secular perturbations of other planets did not change the final resonance state.

The results of the numerical experiments, including the tidal perturbation caused by the other planets, showed us 
that even being a close-packed system, the gravitational effect of other members did not cause any change in the final resonance and the probability of capture is practically the same. Only in the first case, when we go from a 1 to a 3planets configuration, the probability changes for the $3: 2$ and 2:1 resonance. In the other case, when we went to the 6 -planets configuration the probability did not change at all.

The presence of more planets only shows a little effect on the oscillation around the resonance at the end of the spin-orbit evolution, just before reaching the final resonance, especially for El planet $c$ (see Figure 5).

After analysing the dynamical evolution of 2, 3 and 6 planets (see Section 2), we conclude that this particular system have a secular evolution. There are no variations on the semi-major axis caused by the other planets. Variations on eccentricity are also mainly secular.

We conclude that the gravitational influence of other planets is negligible for the spin-down times and for the probabilities of capture in low resonances.

In respect to the stellar system, it is a triple-star system, where the main pair of the system GJ 667A and GJ $667 \mathrm{~B}$ are 0.73 and $0.69 M_{\odot}$ respectively. The pair is separated by $12.6 \mathrm{au}$ between them and $230 \mathrm{au}$ from GJ 667C.

With the purpose of figuring out the actual influence of the binary pair over the dynamical evolution of the system around star C, we developed numerical experiments including $\mathrm{AB}$ stars and the $\mathrm{C}$ star with planet $c$. The star-planet system orbits the center of mass of the binary. We let the system evolve during a period higher than the orbital period of the C star.

Although pair $\mathrm{AB}$, the orbit of star $\mathrm{C}$ and the orbit of planet $c$ around this one, did not suffer any changes at all. Even when the system C-c is close to the periapsis of the orbit, $\sim 180 A U$, the orbit of the planet did not suffer any changes in its evolution.

It should be noted that the orbital periods of the planets around star $\mathrm{C}$ are a lot less than the period of the star around the binary. The period of the C-star is in the order of centuries. On the other hand, the period of the binary is 42.15 years, far from the main frequency of the secular variations of the eccentricity, that is close to 17 years.

\subsection{Capture Probabilities in low Resonances}

The most stable resonance is 3:2 for an El composition. This is an interesting result if we think about the distribution of heat around the surface of the planet. There are recent studies dedicated to the investigation of the photosynthetic potential of planets in 3:2 spin-orbit resonances (Brown et al. 2014). The probability of reaching a 3:2 resonance, in most cases, appears to be significant.

Composition has an effect on the capture probabilities, decreasing the probability for resonances $2: 1$ and 3:2 to zero for a $\mathrm{Ww}$ composition. Although, this looks contradicts the classical idea, due to the dissipation of energy, our results show that $\mathrm{Ww}$ planets dissipated tidal heat faster than the El planets. The result of the spin-down time can be explained by the larger size of the water-rich planet.

\subsection{Habitability of GJ 667C}

Previous works propose the possible location of three planets $(c, f$ and $e$ ) or maybe four $(d)$ inside the HZ (AngladaEscudé et al. 2013). They suggested that planet $d$ could be a water-rich world, making it more habitable. Taking into account the average mass of the planets and the packed configuration of the system, they proposed the formation place beyond the snow-line and a consequent migration to their current positions close to the star. This makes the planets in GJ 667C volatile-rich planets containing substantial amounts of water.

On the other hand, Makarov \& Berghea (2014) dismissed the possible habitability of planet $c$ based on their results of the spin-orbit evolution and the tidal heating of the interior, which makes this planet more similar to Mercury than Earth. Based on our own results, we can state the following about the habitability in GJ 667C.

\subsubsection{Planet $b$}

This planet is obviously beyond the inner limit of the HZ, this makes it not habitable at all. The final rotation period reached, 4.8 days. This period places this super-Earth in a slow rotators category according to the classification of Zuluaga \& Cuartas (2012). Its mass of $5.94 M_{\oplus}$ is unfauvorable when thinking about the possible generation of a protective magnetic field (Zuluaga et al. 2013).

\subsubsection{Planet $c$}

Its distance to the star places it inside the HZ. With a final rotation period of 18.75 days and a mass of $3.86 M_{\oplus}$, this super-Earth is classified as a very slow rotator (Zuluaga \& Cuartas 2012). This means that if planet $c$ had an active magnetic field, the dynamo would be shut down by now. The absence of a protective magnetic field directly leads to an irreversible volatile loss. If at any point in the past, planet $c$ had water on its surface, it no longer exists. This implies that planet $c$ is no longer a habitable planet, even if it was in the distant past.

If we think about the 6-planets configuration, where most of the planets in GJ 667C finally reach a low resonance, even those inside the HZ, they will bear the aggressive activity of their host star during extended periods of time on their diurnal hemispheres. Their atmospheres (if they have any) would be eroded, causing the loss of volatiles, like water, in a time shorter than necessary for life's chemistry to take place.

Finally, as a sideline conclusion, the difference between the capture probabilities, giveb by composition, could be useful in a future as a tool to recognize the actual composition of low mass exoplanets orbiting M-dwarfs, provided that its rotation is feasible to be measured. Earth-like planets seem more probable to be in a 3:2 resonance than Waterrich planets. 


\section{ACKNOWLEDGEMENTS}

We thank the referee for the valuable insights and comments, all of them have been included in the final version of the text. We want to thank Sebastian Bustamante for helping us with the calculation of the interior structure of water-rich planets. We appreciate the revision of the first version of the text made by Esteban Silva-Villa. We are very grateful with Michael Efroimsky and Wade Garret Henning for their useful discussion about some of the structural issues of this paper. We appreciate the careful revision of the final version of the manuscript made by Michael Efroimsky, Julien Frouard and Benoît Noyelles, their suggestions were very useful to us. FACom group is supported by Estrategia de Sostenibilidad 2015-2016, Vicerectora de Investigacin UdeA and by CODI-UdeA project: IN634CE. Mario Melita is supported by CONICET-IAFE, Argentina.

\section{REFERENCES}

Adams E. R., Seager S., Elkins-Tanton L., 2008, ApJ , 673, 1160

Anglada-Escudé G. et al., 2012, ApJL , 751, L16

Anglada-Escudé G. et al., 2013, A\&A , 556, A126

Barnes R., Mullins K., Goldblatt C., Meadows V. S., Kasting J. F., Heller R., 2012, in AAS/Division of Dynamical Astronomy Meeting, Vol. 43, AAS/Division of Dynamical Astronomy Meeting, p. 02.06

Batygin K., Laughlin G., Meschiari S., Rivera E., Vogt S., Butler P., 2009, ApJ , 699, 23

Benneke B., Seager S., 2013, ApJ , 778, 153

Bolmont E., Raymond S. N., Leconte J., Hersant F., Correia A. C. M., 2015, A\&A , 583, A116

Bonfils X. et al., 2013, A\&A , 556, A110

Brown S. P., Mead A. J., Forgan D. H., Raven J. A., Cockell C. S., 2014, International Journal of Astrobiology, 13, 279

Callegari N., Rodríguez Á., 2013, Celestial Mechanics and Dynamical Astronomy, 116, 389

Cayrel de Strobel G., 1981, Bulletin d'Information du Centre de Donnees Stellaires, 20, 28

Correia A. C. M., Boué G., Laskar J., Rodríguez A., 2014, A\&A , 571, A50

Correia A. C. M. et al., 2010, A\&A , 511, A21

Correia A. C. M., Laskar J., 2001, Nature , 411, 767

Correia A. C. M., Laskar J., 2004, Nature , 429, 848

Correia A. C. M., Levrard B., Laskar J., 2008, A\&A , 488, L63

Danby J., 1962, Fundamentals of celestial mechanics

Darwin G. H., 1879, The Observatory, 3, 79

Efroimsky M., 2012, Celestial Mechanics and Dynamical Astronomy, 112, 283

Efroimsky M., Lainey V., 2007, Journal of Geophysical Research (Planets), 112, 12003

Efroimsky M., Makarov V. V., 2013, ApJ , 764, 26

Efroimsky M., Williams J. G., 2009, Celestial Mechanics and Dynamical Astronomy, 104, 257

Feroz F., Hobson M. P., 2014, MNRAS , 437, 3540

Ferraz-Mello S., 2013, Celestial Mechanics and Dynamical Astronomy, 116, 109
Ferraz-Mello S., Rodríguez A., Hussmann H., 2008, Celestial Mechanics and Dynamical Astronomy, 101, 171

Giacaglia G. E. O., 1976, Celestial Mechanics, 14, 515

Gladman B., Quinn D. D., Nicholson P., Rand R., 1996, Icarus , 122, 166

Goldreich P., 1966, AJ , 71, 1

Goldreich P., Peale S., 1966, AJ , 71, 425

Goldreich P., Peale S. J., 1968, ARA\&A , 6, 287

Heller R., Barnes R., Leconte J., 2011, Origins of Life and Evolution of the Biosphere, 37

Henning W. G., O'Connell R. J., Sasselov D. D., 2009, ApJ , 707, 1000

Hut P., 1981, A\&A , 99, 126

Kaltenegger L., Sasselov D., Rugheimer S., 2013, ApJL , 775, L47

Kaula W. M., 1961, Geophysical Journal, 5, 104

Kaula W. M., 1964, Reviews of Geophysics and Space Physics, 2, 661

Kuchner M. J., 2003, ApJL , 596, L105

Lammer H. et al., 2010, Astrobiology, 10, 45

Laskar J., 1988, A\&A , 198, 341

Laskar J., Boué G., Correia A. C. M., 2012, A\&A , 538, A105

Leconte J., Chabrier G., Baraffe I., Levrard B., 2010, A\&A , 516, A64

Léger A. et al., 2004, Icarus , 169, 499

Lomb N. R., 1976, Ap\&SS , 39, 447

MacDonald G. J. F., 1964, Reviews of Geophysics and Space Physics, 2, 467

Makarov V. V., 2012, ApJ , 752, 73

Makarov V. V., 2015, ApJ , 810, 12

Makarov V. V., Berghea C., 2014, ApJ , 780, 124

Makarov V. V., Berghea C., Efroimsky M., 2012, ApJ , 761, 83

Makarov V. V., Efroimsky M., 2013, ApJ , 764, 27

Mardling R. A., 2007, MNRAS , 382, 1768

Moore W. B., Schubert G., 2003, Icarus , 166, 223

Murray C. D., Dermott S. F., 1999, Solar system dynamics Rodríguez A., Callegari, Jr N., Michtchenko T. A., Hussmann H., 2012, ArXiv e-prints

Schaefer L., Sasselov D., 2015, ApJ , 801, 40

Vance S., Bouffard M., Choukroun M., Sotin C., 2014, Planet. Space Sci. , 96, 62

Williams J. G., Efroimsky M., 2012, Celestial Mechanics and Dynamical Astronomy, 114, 387

Wu Y., Goldreich P., 2002, ApJ , 564, 1024

Zechmeister M., Kürster M., 2009, A\&A , 496, 577

Zuluaga J. I., Bustamante S., Cuartas P. A., Hoyos J. H., 2013, ApJ , 770, 23

Zuluaga J. I., Cuartas P. A., 2012, Icarus , 217, 88 